\title{
Invited Discussion on: Individualized Surgical Treatment of Different Types of Labia Minora Hypertrophy
}

\author{
Lina Triana ${ }^{1}(D)$
}

Received: 11 November 2019/Accepted: 17 November 2019/Published online: 2 January 2020

(C) Springer Science+Business Media, LLC, part of Springer Nature and International Society of Aesthetic Plastic Surgery 2020

Level of Evidence $V$ This journal requires that authors assign a level of evidence to each article. For a full description of these Evidence-Based Medicine ratings, please refer to the Table of Contents or the online Instructions to Authors www.springer.com/00266.

First of all, I want to congratulate the authors for bringing once more to our Aesthetic Plastic Surgery journal a subject related to vagina plastic surgery that according to ISAPS statistics has increased more than any other aesthetic plastic surgery procedure in the last 2 years.

The vascular anatomy could have been better described at the beginning of the article because large resections of the labia minora are proposed in these surgical methods. Although the labia minora have good vascularization, because the proposed techniques include long flaps, a better schematic description of these vessels would have justified safety on the flap designs and overall would have enriched the paper.

More deliberation on why the authors decided to do these modified wedge resections would have been important to bring up on the paper. It would be helpful to compare them with the traditional wedge and edge resections and why they believe they can give better results, pros and cons, for more assertive communication with the reader on why to incorporate into their practice their proposed labia minora resections.

Also, better drawings on each technique can facilitate others to correctly reproduce the technique. A better description and step-by-step surgical technique is a must when describing new options or modifications of surgical

Lina Triana

linatriana@drlinatriana.com

1 Clinica Corpus Y Rostrum, Cali, Valle, Colombia technique, and this paper certainly lacks these for each different resection that they propose.

A good point is how they comment that every patient must be addressed as unique, and by saying so, each surgery plan too. The anatomy of the genital area varies from patient to patient, and this is why no two surgical plans can be the same. Also, when we refer to vagina and genital aesthetics we have no molds, no two patients are the same and no two patients perceive their vagina in the same way.

This variety in the shape and how every woman feels about their genitals must be always present in the mind of the plastic surgeon. When performing labiaplasties, because no two women are the same, surgeon must have a conceptual grasp of the whole area, not only the labia minora, to be able to address all the areas and not just one structure. For example, if the patient has an important excess of clitoris hood and nothing is done to it, only resection of the labia minora is done, many times the patient will end up unhappier than how she was before having the labiaplasty done. Many will even say now that this excess of their clitoris hood makes them look like they have a penis. So never hesitate in taking away excess mucosa of the clitoris hood when present.

Another important aspect that the authors addressed very well in the article is that as surgeons we need to have many surgical options to treat labia minora because they are so variable in appearance. And this is one of the main reasons the authors describe these four different options to address labia minora hypertrophy, based on where the excess is present, in which location of the labia minora, and according to this excess the resection is be planned.

Although the authors justify their technique by saying that the labia minora are well vascularized, I still believe the technique is pretty aggressive leaving a flap that is too 
long in proportion with its base. It surprised me even more when in the discussion the authors mention that if a wedge resection method is chosen, the length and width of the flap should not exceed 1:1 and that gentle and minimal invasion should be done to these labia to avoid flap necrosis. With these long flaps proposed by the authors, it surprised me that necrosis of flap tips was low.

One of the more valuable ideas that are presented with this technique and that would have been worth discussing more in the article would have been to expand the concept of the labia minora edge darkness and how they are able to address it resolving two of the main concerns described by the two most popular labiaplasty resection methods, the wedge and edge resections.

One of the big benefits of a wedge labiaplasty resection is that the labia minora border is preserved, but by doing so, the dark color, that many patients hate, is preserved, too. On the contrary, with edge labiaplasty resection the dark color is resolved but many times the technique is criticized because it can have an irregular and unnatural labia minora border afterward.

This wedge resection with long flaps proposed by the authors of this paper seems to resolve both the color and edge appearance situations because the resection is done where the thick and/or dark color is present and the long flap will be left as the natural labia border, once again resolving both problems, color and leaving a natural border, at once.
This is an interesting paper that once again brings to all of those surgeons wanting to do vaginal plastic surgery procedures the importance of addressing each patient as unique, not only because of the unique nature of the genital anatomy, no two patients are the same, but also from the unique aspect that every woman perceives her vagina in a different way.

Welcomed are all modifications to labiaplasty and even more if they can help us resolve the differences in color and morphology of labia minora with specific kinds of surgical methods that improve our results in this procedure and increases every day. A labiaplasty is done for much more than just functional or aesthetic reasons; vagina plastic surgery procedures are here to empower woman with their sexuality bringing a woman one step forward in leading all aspects of her life.

\section{Compliance with Ethical Standards}

Conflict of interest The authors declare that they have no conflicts of interest to disclose.

Human and animal rights This article does not contain any studies with human participants or animals performed by any of the authors.

Informed consent For this type of study informed consent is not required.

Publisher's Note Springer Nature remains neutral with regard to jurisdictional claims in published maps and institutional affiliations. 